\title{
Alternative method to characterize continuous and discontinuous phase transitions in surface reaction models
}

\author{
Henrique A. Fernandes, ${ }^{1}$ Roberto da Silva, ${ }^{2}$ Eder D. Santos, ${ }^{1}$ Paulo F. Gomes, ${ }^{1}$ and Everaldo Arashiro ${ }^{3}$ \\ ${ }^{1}$ Coordenação de Física, Universidade Federal de Goiás, Regional Jataí, BR 364, km 192, 3800-CEP 75801-615, \\ Jataí, Goiás, Brazil \\ ${ }^{2}$ Instituto de Física, Universidade Federal do Rio Grande do Sul, Av. Bento Gonçalves, 9500-CEP 91501-970, Porto Alegre, \\ Rio Grande do Sul, Brazil \\ ${ }^{3}$ Instituto de Matemática, Estatística e Física, Universidade Federal do Rio Grande, Campus Carreiros, Av. Itália, km 8-CEP 96203-900, Rio \\ Grande, Rio Grande do Sul, Brazil
}

(Received 2 July 2016; published 22 August 2016)

\begin{abstract}
In this paper we revisited the Ziff-Gulari-Barshad model to study its phase transitions and critical exponents through time-dependent Monte Carlo simulations. We use a method proposed recently to locate the nonequilibrium second-order phase transitions and that has been successfully used in systems with defined Hamiltonians and with absorbing states. This method, which is based on optimization of the coefficient of determination of the order parameter, was able to characterize the continuous phase transition of the model, as well as its upper spinodal point, a pseudocritical point located near the discontinuous phase transition. The static critical exponents $\beta, v_{\|}$, and $v_{\perp}$, as well as the dynamic critical exponents $\theta$ and $z$ for the continuous transition point, were also estimated and are in excellent agreement with results found in literature.
\end{abstract}

DOI: 10.1103/PhysRevE.94.022129

\section{INTRODUCTION}

In recent years, the study of kinetic or nonequilibrium systems [1,2] has grown considerably, making them a fruitful subject in some branches of the biological [3,4], financial [5,6], social $[7,8]$, and applied sciences $[9,10]$. Major efforts and interest have been put on systems which exhibit nonequilibrium phase transitions and critical phenomena such as transport phenomena, traffic jams, and epidemic spreading [11]. In this context, one can also consider the directed percolation (DP) [12]: an important case of nonequilibrium critical phenomena whose class cover other interesting models with universal exponents. Systems belonging to DP universality class exhibit a continuous phase transition from an active phase to an absorbing phase. The absorbing phase represents states in which, once reached, the systems become trapped and can not escape. As conjectured by Janssen [13] and Grassberger [14] there exist many physical systems belonging to the DP universality class. Nevertheless, experimental observations of such a behavior have not been shown frequently in the literature.

Other nonequilibrium systems which present phase transitions and critical phenomena are related to surface reaction models [15-18]. In fact, these models have attracted considerable interest, whereas they can be used to explain several experimental observations in catalysis [19-21]. For instance, in 1986, Ziff, Gulari, and Barshad [22] devised a stochastic model that describes some nonequilibrium aspects of the catalytic reaction of carbon monoxide and oxygen to produce carbon dioxide $\left(\mathrm{CO}+\mathrm{O} \rightarrow \mathrm{CO}_{2}\right)$ on a surface and that, in addition, exhibits continuous and discontinuous phase transitions. Several works have shown that its critical point belongs to the DP universality class [23]. Due to its simplicity, rich phase diagram, and experimental observation of the discontinuous phase transition, the Ziff-Gulari-Barshad model, also known as the ZGB model, has become a prototype for the study of reaction processes on catalytic surfaces [24-26].

After its advent, a number of authors have proposed some modified versions of the ZGB model in order to obtain more realistic systems of actual catalytic processes. For instance, it was modified to include $\mathrm{CO}$ desorption [26-31], diffusion [19,26,30-32], impurities [33-37], attractive and repulsive interactions between the adsorbed molecules [38], surfaces of different geometries [24,39] and with hard oxygen boundary conditions [40], etc. In addition, it has been studied through several techniques, such as simulations, mean-field theories, series analysis, etc [41].

In this paper, we revisit the ZGB model as proposed in Ref. [22] in order to study its phase transitions and critical exponents by using short-time dynamics. By considering timedependent Monte Carlo simulations and a nonconventional optimization method, based on a simple statistical concept known as coefficient of determination (see, for example, Ref. [42]), we were able to refine the continuous transition point and, surprisingly, obtain an accurate estimate to the upper spinodal point associated to the discontinuous transition point. This technique has been used in the study of reversible systems [43-46] and was considered recently in the study of an epidemic model to determine its critical immunization probability [47].

The paper is organized as follows: In the next section, we present the model, and in Sec. III we describe the short-time Monte Carlo simulation technique as well as the coefficient of determination. In Sec. IV we show our main numerical results and illustrations. Finally, a brief summary is presented in Sec. V.

\section{ZIFF-GULARI-BARSHAD MODEL}

The Ziff-Gulari-Barshad (ZGB) model [22] is a dimermonomer lattice model which simulates the catalysis between 
the carbon monoxide $(\mathrm{CO})$ and the oxygen molecule $\left(\mathrm{O}_{2}\right)$. The reactions follow the Langmuir-Hinshelwood mechanism $[22,48]$ and are summarized in three steps:

$$
\begin{gathered}
\mathrm{CO}(g)+V \rightarrow \mathrm{CO}(a), \\
\mathrm{O}_{2}(g)+2 V \rightarrow 2 \mathrm{O}(a), \\
\mathrm{CO}(a)+\mathrm{O}(a) \rightarrow \mathrm{CO}_{2}(g)+2 V,
\end{gathered}
$$

where $g$ and $a$ refer, respectively, to the gas and adsorbed phases of the atoms and molecules, $\mathrm{CO}_{2}$ stands for carbon dioxide molecule, and $V$ means a vacant site on the surface.

Computationally, this catalytic surface can be modeled as a regular square lattice and its sites might be occupied by $\mathrm{CO}$ molecules or by oxygen $(\mathrm{O})$ atoms or may be empty. By using the Monte Carlo method, the simulation is carried out as follows [22,41,49]: In the gas phase, the CO molecule is chosen to impinge on the surface at rate $y_{\mathrm{CO}}$, while the $\mathrm{O}_{2}$ molecule strikes the lattice at rate $y_{\mathrm{O}_{2}}=1-y_{\mathrm{CO}}$. As these rates are relative ones and $y_{\mathrm{CO}}+y_{\mathrm{O}_{2}}=1$, the model has a single free parameter: $y=y_{\mathrm{CO}}$. According to Eq. (1), if the $\mathrm{CO}$ molecule is selected in the gas phase, a site on the surface is chosen at random and, if it is vacant $(V)$, the molecule is adsorbed on this site. Otherwise, if the chosen site is occupied by a CO molecule or by an $\mathrm{O}$ atom, the trial ends, the $\mathrm{CO}$ molecule returns to the gas phase, and a new molecule is chosen. However, if the $\mathrm{O}_{2}$ molecule is selected, then a nearest-neighbor pair of sites is chosen at random. If both sites are vacant, the $\mathrm{O}_{2}$ molecule dissociates into a pair of $\mathrm{O}$ atoms which are adsorbed on the chosen lattice sites [Eq. (2)]. Otherwise, if one or both sites are occupied, the trial ends, the $\mathrm{O}_{2}$ molecule returns to the gas phase, and a new molecule is chosen. Equation (3) stands for the reaction between the $\mathrm{CO}$ molecule and the $\mathrm{O}$ atom, both adsorbed on the lattice. Immediately after each adsorption event, the nearest-neighbor sites of the adsorbed molecule are checked. If a O-CO pair is found, the $\mathrm{CO}_{2}$ molecule is formed and quits the lattice, leaving two vacant sites on it. However, if there is the formation of two or more O-CO pairs, a pair is chosen at random to quit the lattice.

The ZGB model has been extensively studied and nowadays is considered a prototype for the study of reaction processes on catalytic surfaces. This is mainly due to its simplicity and rich phase diagram with three distinct steady-state phases separated by continuous and discontinuous phase transitions [22,24-26]. For $0<y<y_{1}$ the surface becomes irreversibly poisoned (saturated) by $\mathrm{O}$ atoms (O-poisoned state). At $y=y_{1} \cong$ 0.3874 [50] there is a continuous phase transition from the $\mathrm{O}$-poisoned state to an active phase where there is sustainable production of $\mathrm{CO}_{2}$ molecules. This state ends when $y=y_{2} \cong$ 0.5256 [51], whereas for this point the system undergoes a discontinuous phase transition and the surface becomes irreversibly poisoned by $\mathrm{CO}$ molecules (CO-poisoned state). For $y_{2}<y \leqslant 1$ the surface remains in the CO-poisoned state, i.e., every site on the surface is occupied by CO. In summary, $y_{1}$ and $y_{2}$ are irreversible phase transition (IPT) points between the reactive and poisoned states. While $y_{1}$ is related to the continuous IPT, $y_{2}$ represents the discontinuous one. Although some experimental works on platinum confirm the existence of discontinuous transition in the catalytic oxidation of $\mathrm{CO}$
[19,20,52-55], there is no experimental evidence of continuous IPT despite its existence in the theoretical framework. In this case it is well established that this transition belongs to the DP universality class $[23,56]$.

\section{FINITE SIZE SCALING AND TIME-DEPENDENT MONTE CARLO SIMULATIONS}

The finite size scaling near criticality of systems belonging to the DP universality class can be described by

$$
\langle\rho(t)\rangle \sim t^{-\beta / v_{\|}} f\left(\left(y-y_{c}\right) t^{1 / v_{\|}}, t^{d / z} L^{-d}, \rho_{0} t^{\beta / v_{\|}+\theta}\right),
$$

where $\langle\cdots\rangle$ means the average on different evolutions of the system, $d$ is the dimension of the system ( $d=2$ for the ZGB model), $L$ is its linear size, and $t$ is the time. The exponents $z=v_{\|} / \nu_{\perp}$ and $\theta=\frac{d}{z}-\frac{2 \beta}{\nu_{\|}}$are dynamic critical exponents, and $\beta, v_{\|}$, and $v_{\perp}$ are static ones. Here $y-y_{c}$ denotes the distance of a point $y$ to the critical one point, $y_{c}$, which governs the algebraic behaviors of the two independent correlation lengths: the spatial one which behaves as $\xi_{\perp} \sim\left(y-y_{c}\right)^{-\nu_{\perp}}$ and the temporal one, $\xi_{\|} \sim\left(y-y_{c}\right)^{-\nu_{\|}}$. Basically, $\xi_{\perp}$ must be thought of as the average over many independent realizations of the cluster diameter while $\xi_{\|}$is the same average of the required time to reach the absorbing state. Besides the density of $\mathrm{CO}$ molecules $\rho_{\mathrm{CO}}$, one can also consider the density of empty (vacant) sites $\rho_{V}$ as the order parameter of the model. Therefore, in Eq. (4), $\rho$ stands for a generic density which can be $\rho_{\mathrm{CO}}$ or $\rho_{V}$. The density is given by

$$
\rho(t)=\frac{1}{L^{d}} \sum_{j=1}^{L^{d}} s_{j} .
$$

According to the density which is taken into consideration, $s_{j}=1$ when the sites $j$ are occupied by CO molecules (for $\rho_{\mathrm{CO}}$ ) or when they are vacant (for $\rho_{V}$ ). Otherwise, the $s_{j}=0$. As can be seen in Sec. IV, part of our results are obtained by considering both order parameters.

The dynamic and static critical exponents of the model can be obtained by using Eq. (4) and performing time-dependent Monte Carlo simulations with two different initial conditions. Equation (4) can be observed in another way:

$$
\langle\rho\rangle\left(t, L, \rho_{0}\right)=L^{-\beta / \nu_{\perp}}\langle\rho\rangle\left(L^{-z} t, L^{x_{0}} \rho_{0}\right),
$$

where $x_{0}=\beta / v_{\perp}+z \theta$ at $y=y_{c}$. Denoting $u=t L^{-z}$ and $w=L^{x_{0}} \rho_{0}$, the derivative with respect to $L$ gives

$$
\begin{aligned}
\partial_{L}\langle\rho\rangle= & \left(-\beta / v_{\perp}\right) L^{-\beta / \nu_{\perp}-1}\langle\rho\rangle(u, w) \\
& +L^{-\beta / \nu_{\perp}}\left[\partial_{u}\langle\rho\rangle \partial_{L} u+\partial_{w}\langle\rho\rangle \partial_{L} w\right],
\end{aligned}
$$

where we have explicitly $\partial_{L} u=-z t L^{-z-1}$ and $\partial_{L} w=$ $x_{0} \rho_{0} L^{x_{0}-1}$. In the limit $L \rightarrow \infty$, which implies $\partial_{L}\langle\rho\rangle \rightarrow 0$, one has $x_{0} w \partial_{w}\langle\rho\rangle-z u \partial_{u}\langle\rho\rangle-\beta / \nu_{\perp}\langle\rho\rangle=0$. The separability of the variables $u$ and $w$, i.e., $\langle\rho\rangle(u, w)=\langle\rho\rangle_{u}(u)\langle\rho\rangle_{w}(w)$, leads to

$$
x_{0} w\langle\rho\rangle_{w}^{\prime} /\langle\rho\rangle_{w}=\beta / \nu_{\perp}+z u\langle\rho\rangle_{u}^{\prime} /\langle\rho\rangle_{u}=c,
$$

where $c$ must be equal a constant. So we have $\langle\rho\rangle_{u}=u^{c / z}-$ $\beta /\left(v_{\perp} z\right)$ and $\langle\rho\rangle_{w}=w^{c / x_{0}}$, which leads to

$$
\langle\rho\rangle(t)=\rho_{0}^{c / x_{0}} t^{\left(c-\beta / v_{\perp}\right) / z} .
$$


When one considers the system starting with all sites empty, there is no dependence on initial conditions $(c=0)$ and

$$
\langle\rho\rangle(t) \sim t^{-\beta / \nu_{\|}} .
$$

However, when the simulation starts with all sites of the lattice filled with $\mathrm{O}$ atoms but a random site which remains empty, we can choose $c=x_{0}$, which leads to

$$
\langle\rho\rangle(t) \sim \rho_{0} t^{\theta}=\rho_{0} t^{\left(\frac{d}{z}-2 \frac{\beta}{v_{\|}}\right)} .
$$

Here it is important to notice an interesting crossover phenomenon [2]. By starting with an initial density $\rho_{0}$, the density of active sites (empty sites in our case) increases as shown in Eq. (6). This phenomenon is known as the critical initial slip of nonequilibrium systems and occurs until it reaches a maximum value at time $t_{\max }$. Thereafter, the system crosses over to the usual relaxation described by the power law decay given by Eq. (5). In summary,

$$
\langle\rho\rangle(t)=\left\{\begin{array}{ll}
\rho_{0} t^{\theta} & \text { if } t<t_{\max } \\
t^{-\beta / \nu_{\|}} & \text {for } t>t_{\max }
\end{array},\right.
$$

where $t_{\max }$ is the solution of $\rho_{0} t_{\max }^{\theta}=t_{\max }^{-\beta / \nu_{\|}}$, which gives $t_{\max }=\rho_{0}^{-1 /\left(\frac{d}{z}-\frac{\beta}{v_{\|}}\right)}$. Such a relaxation is similar to that one which occurs for spin systems when they are quenched from high temperature to the critical one [57].

An interesting manner to obtain the exponent $z$ from an independent way is to combine simulations with different initial conditions. This idea has been applied successfully in a large number of spin systems: for example, the Ising model, the $q=3$ and $q=4$ Potts models [58] and the Heisenberg model [59] and even for models based on the generalized Tsallis statistics [43]. It was also introduced recently in systems without defined Hamiltonian, as can be seen in Ref. [60]. To obtain the power law, we consider the cumulant as follows:

$$
F_{2}(t)=\frac{\langle\rho\rangle_{\rho_{0}=1 / L}(t)}{\langle\rho\rangle_{\rho_{0}=1}^{2}(t)} \sim t^{d / z} .
$$

So, once the dimension $d$ of the system is known, a log-log fit of $F_{2}(t) \times t$ yields the exponent $z$.

In addition to the exponents $z$ and $\theta$ which are obtained independently from Eqs. (6) and (7), we can obtain the static critical exponents $\beta, v_{\|}$, and $\nu_{\perp}$ by using the method proposed by Grassberger and Zhang [61] to estimate the exponent $v_{\|}$for DP and used by da Silva et al. [60] to study the one-dimensional contact process and Domany-Kinzel cellular automaton through short-time Monte Carlo simulations:

$$
D(t)=\left.\frac{\partial \ln \langle\rho\rangle}{\partial y}\right|_{y=y_{c}}=t^{\frac{1}{\|\|}}
$$

Here the derivative is numerically represented by

$$
D(t)=\frac{1}{2 \delta} \ln \left[\frac{\langle\rho\rangle\left(y_{c}+\delta\right)}{\langle\rho\rangle\left(y_{c}-\delta\right)}\right],
$$

where $\delta$ is a tiny perturbation needed to move the system slightly off the criticality.

\section{RESULTS}

Nonequilibrium Monte Carlo simulations were first designed to study second-order critical points, whereas at these points universality and scaling behavior is observed even at the early stages of time evolution [57,62]. However, it has been shown that this technique is also important in the study of weak first-order phase transitions $[63,64]$ since these transitions possess long correlation lengths and small discontinuities and therefore behave similarly to second-order phase transitions. It has been conjectured that near a weak first-order transition there exist two pseudocritical points: one point is just below (inferior) the first-order point, and the other is just above (upper) it. These pseudocritical points are known as spinodal points.

In this contribution, we divide our results in two parts. First, we perform nonequilibrium Monte Carlo simulations to characterize the continuous and discontinuous phase transitions of the model. For this task, we use an alternative method based on optimization of the coefficient of determination of power laws. Surprisingly, we obtain a description of the upper spinodal point, which has not been observed by this method, developed by one of authors [43], for models without defined Hamiltonian.

In the second part of our results, we carry out shorttime Monte Carlo simulations to determine the static critical exponents $\beta, v_{\|}, v_{\perp}$, and the dynamic critical exponents $z$ and $\theta$ of the continuous point of the ZGB model using a set of power laws. In this study, we show that the method of mixed initial conditions applied to other models without defined Hamiltonian [60] (contact process, cellular automata) can be adapted also to obtain the dynamic exponent $z$ of the ZGB model.

\section{A. Results I: Exploration of the upper spinodal point and continuous transition point using the coefficient of determination}

The main goal of this work is to study the phase transition points of the ZGB model via time-dependent Monte Carlo (MC) simulations by estimating the best $y$ given as input the parameter $y^{(\mathrm{min})}$ (initial value) and run simulations for different values of $y$ up to $y^{(\max )}$, according to a resolution $\Delta y$.

For this task, we used an approach developed in Ref. [43] in the context of generalized statistics. This tool had also been applied successfully to study multicritical points, for example, tricritical points [45] and Lifshitz point of the ANNNI model [44] and Z5 model [46], and also in models without a defined Hamiltonian [47].

Since at criticality $\left(y=y_{c}\right)$ it is expected that the order parameter obeys the power law behavior of Eq. (5), we performed MC simulations for each value of $y=y^{(\min )}+$ $i \Delta y$, with $i=1, \ldots, n$, where $n=\left\lfloor\left(y^{(\max )}-y^{(\min )}\right) / \Delta y\right\rfloor$, and calculated the coefficient of determination, which is given by

$$
r=\frac{\sum_{t=1}^{N_{M C}}(\overline{\ln \langle\rho\rangle}-a-b \ln t)^{2}}{\sum_{t=1}^{N_{M C}}(\overline{\ln \langle\rho\rangle}-\ln \langle\rho\rangle(t))^{2}},
$$




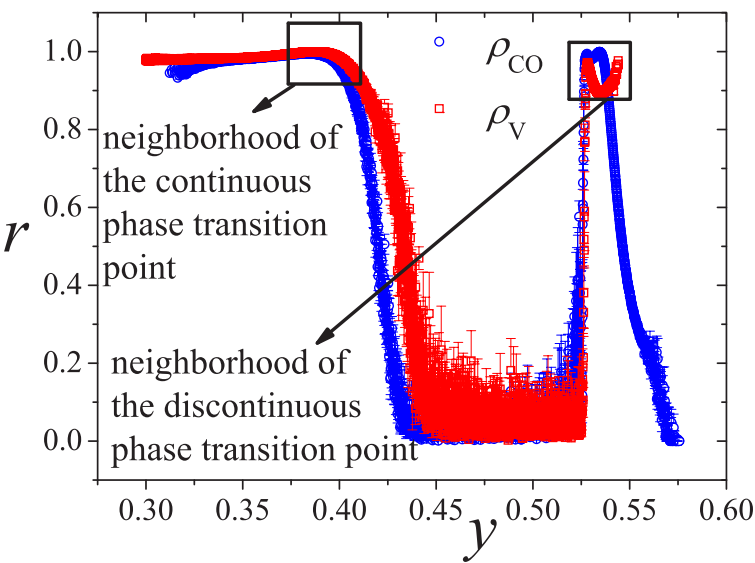

FIG. 1. Coefficient of determination $r$ as function of $y$. The maximum occurs at the expected critical point and in a region related to the discontinuous phase transition, which is also observed in our study. Both regions deserve our attention and are explored in this paper.

where $\overline{\ln \langle\rho\rangle}=\left(1 / N_{M C}\right) \sum_{t=1}^{N_{M C}} \ln \langle\rho\rangle(t)$, and the critical value

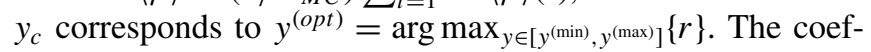
ficient $r$ has a very simple explanation: it measures the ratio (expected variation)/(total variation). The bigger the $r$, the better the linear fit in log scale, and therefore, the better the power law which corresponds to the critical parameter except for an order of error $\Delta y$.

It is important to mention that the coefficient of determination was obtained by considering two order parameters: the density of $\mathrm{CO}$ molecules $\left(\rho_{\mathrm{CO}}\right)$ and the density of empty sites $\rho_{V}$. Although the former is commonly used, some studies have considered $\rho_{V}$ as order parameter (see Ref. [65]). First, we considered a lattice of linear size $L=160$ and explored the scenery in general by estimating $r$ for different values of $y\left(0.3 \leqslant y \leqslant 0.6\right.$ and $\left.\Delta y=10^{-4}\right)$ (see Fig. 1$)$. The square (red) points represent the coefficient of determination obtained when considering $\rho_{V}$, while the circles (blue) represent the coefficient of determination for $\rho_{\mathrm{CO}}$.

As can be seen in this figure, when one takes into account the density of $\mathrm{CO}$ molecules the curve ends for $y \simeq 0.56$. However, when one considers the density of empty sites, the curve ends for $y \simeq 0.55$. The reason is that for higher values of $y$, one obtains undefined values meaning that there is no power law behavior as observed in second-order (continuous) phase transitions and the slope goes to infinity. We can also observe two candidate regions to have phase transitions $(r \simeq 1)$ : one maximum for the expected critical point $(y \simeq 0.3874)$ and a region related to the discontinuous phase transition $(y \simeq$ $0.525)$. Of course, both regions deserve our attention. Hence, we explore such parts by performing simulations for each region, with $\Delta y=10^{-4}$ and for different lattice sizes $(L=$ $40,80,160,240$, and 320). For each lattice, the process was repeated for five different seeds in order to obtain the error bars.

First, we focused our attention to the candidates to the continuous phase transition point. For each lattice size, we obtain the maximum for different seeds by taking an average. Finally, an extrapolation was performed to take into account
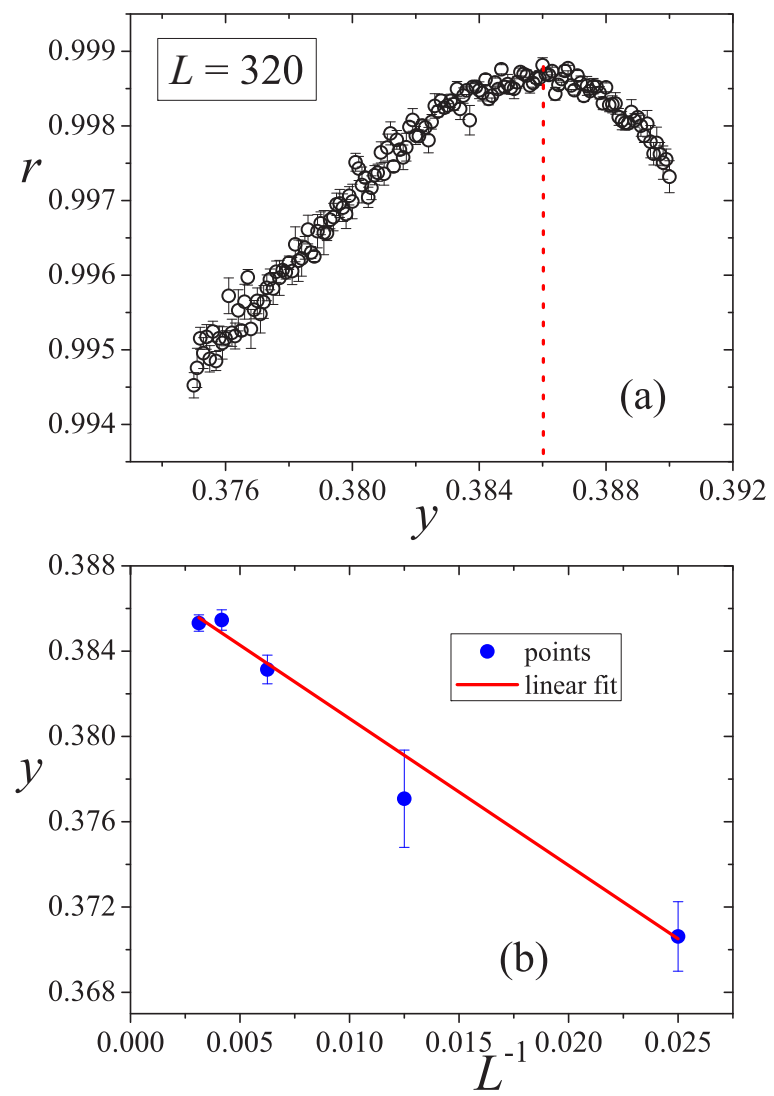

FIG. 2. Determination of continuous phase transition point using the density of $\mathrm{CO}$ molecules through the curve $r \times y$. (a): Localization of the continuous phase transition point for $L=320$. (b) Extrapolation of $y \times 1 / L$ for different lattice sizes used in this paper.

effects of finite size. Figure 2 shows the localization of the continuous phase transition point $y_{1}$ for $\rho_{\mathrm{CO}}$. The behavior of $r$ versus $y$ is shown in Figs. 2(a) and 2(b) presents the extrapolation $y$ versus $1 / L$.

We also study the coefficient of determination considering $\rho_{V}$ as order parameter in order to check the efficiency of the method to obtain the continuous phase transition point of the model. In Fig. 3 we present both behaviors of $r$ versus $y$ for $L=320$ [Fig. 3(a)] and the estimates for different lattice sizes along with the limit procedure [Fig. 3(b)] using the density of vacant sites.

The values for $y_{1}$ (critical point) obtained in this paper are presented in Table I. We can observe an excellent agreement with the first estimate obtained in literature.

TABLE I. Results for the continuous and spinodal points obtained by the method of optimization of power laws. Estimates are in excellent agreement with the literature.

Continuous phase transition point Upper spinodal point

\begin{tabular}{lcl}
\hline$\rho_{\mathrm{CO}}$ & $0.3877(5)$ & $0.52738(14)$ \\
$\rho_{V}$ & $0.3879(2)$ & $0.52764(12)$ \\
Literature & $\cong 0.3874[50]$ & $0.5270(5)[64]$ \\
\hline
\end{tabular}



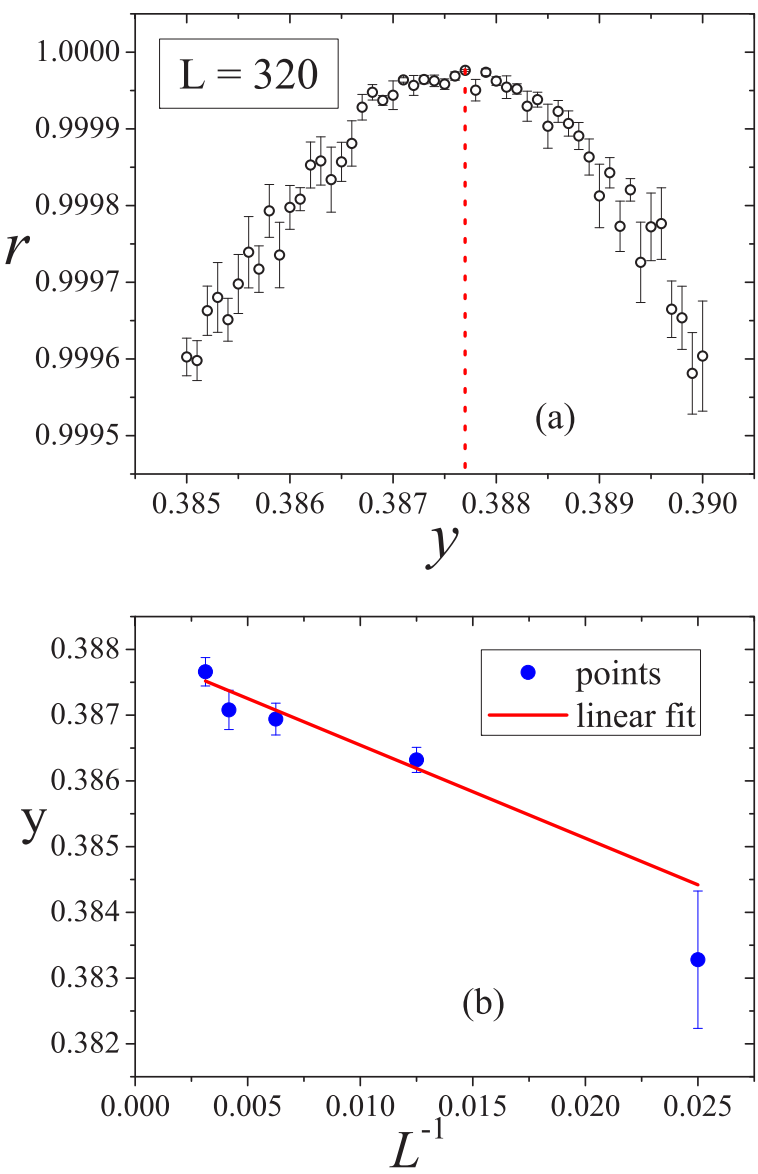

FIG. 3. Determination of continuous phase transition point using the density of empty sites through the curve $r \times y$. (a) Localization of the continuous phase transition point for $L=320$. (b) Limit procedure used to determine $y_{1}$ when $1 / L \rightarrow 0$.

Now, we focus our attention to the upper spinodal point $y_{2}^{u p}$ previously predicted by other authors (see Ref. [64]). Figure 4(a) shows $r \times y$ for the density of $\mathrm{CO}$ molecules for an isolated region which is candidate to contain a weak discontinuous phase transition. This figure presents all lattice sizes considered in this paper. For clarity, the error bars are not shown in the main plots.

In Fig. 4(a) we can see two different "hills." It is important to observe that the second hill does not change for different lattice sizes. Whereas we know that finite size scaling involving discontinuous (first-order) transitions or even their spinodal points (in the case of weak first-order transitions) are notable, we particularly concentrate our attention in the first point, which moves to the left as $L$ increases. Since we have large fluctuations of smaller sizes, we do not use an extrapolation here, and instead we directly calculate the $y$ that maximizes $r$ for five different seeds in our largest lattice $(L=320)$, which is shown in the inset plot in Fig. 4(a) with the appropriated error bars. Surprisingly we find $y_{2}^{u p}=0.52738(14)$, which exactly matches what is found in the literature for the upper spinodal point of the discontinuous transition point [64]. It is expected that spinodal points (also called pseudocritical points) behave as critical points as shown by Schulke and Zheng [63] in
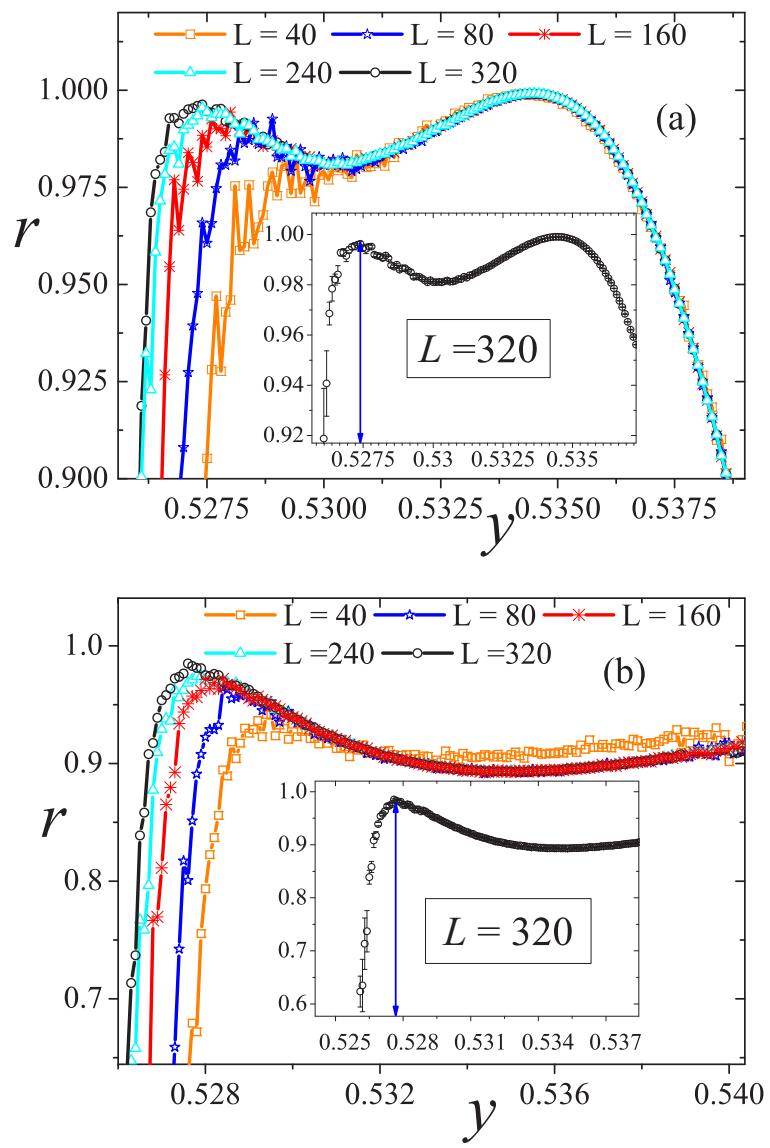

FIG. 4. Determination of the upper spinodal point. (a) Using the density of CO molecules; (b) using the density of empty sites. The plots show the curves $r \times y$ for different lattice sizes. The inset shows the plot only with our larger lattice $L=320$ with error bars indicating the pronounced (first) peak, which corresponds to the upper spinodal point.

the context of short-time dynamics. This excellent agreement led us to investigate the density of empty sites, as shown in Fig. 4(b), and our result, $y_{2}^{u p}=0.52764(12)$, is in excellent agreement with our previous estimate for the upper spinodal point. Our main results are resumed in Table I. Here it is important to mention that the second hill does not appear and its lack of size dependence seems to be correctly disregarded in the first situation.

In the next section we obtain the critical exponents of the critical point.

\section{B. Results II: Critical exponents}

Finally, we perform short-time MC simulations to obtain the dynamic and static critical exponents of the ZGB model. In our simulations, we consider square lattices of linear sizes $L=80,160,240$, and 320 in order to account for finite size effects, and the density of $\mathrm{CO}$ molecules is considered as the order parameter of the model.

Here we considered $N_{M C}=500 \mathrm{MC}$ steps in the study of the time evolution given by Eqs. (5) and (6) and $N_{M C}=1500$ MC steps when considering the Eq. (8). However, first $100 \mathrm{MC}$ steps were disregarded in the calculation of the exponents 

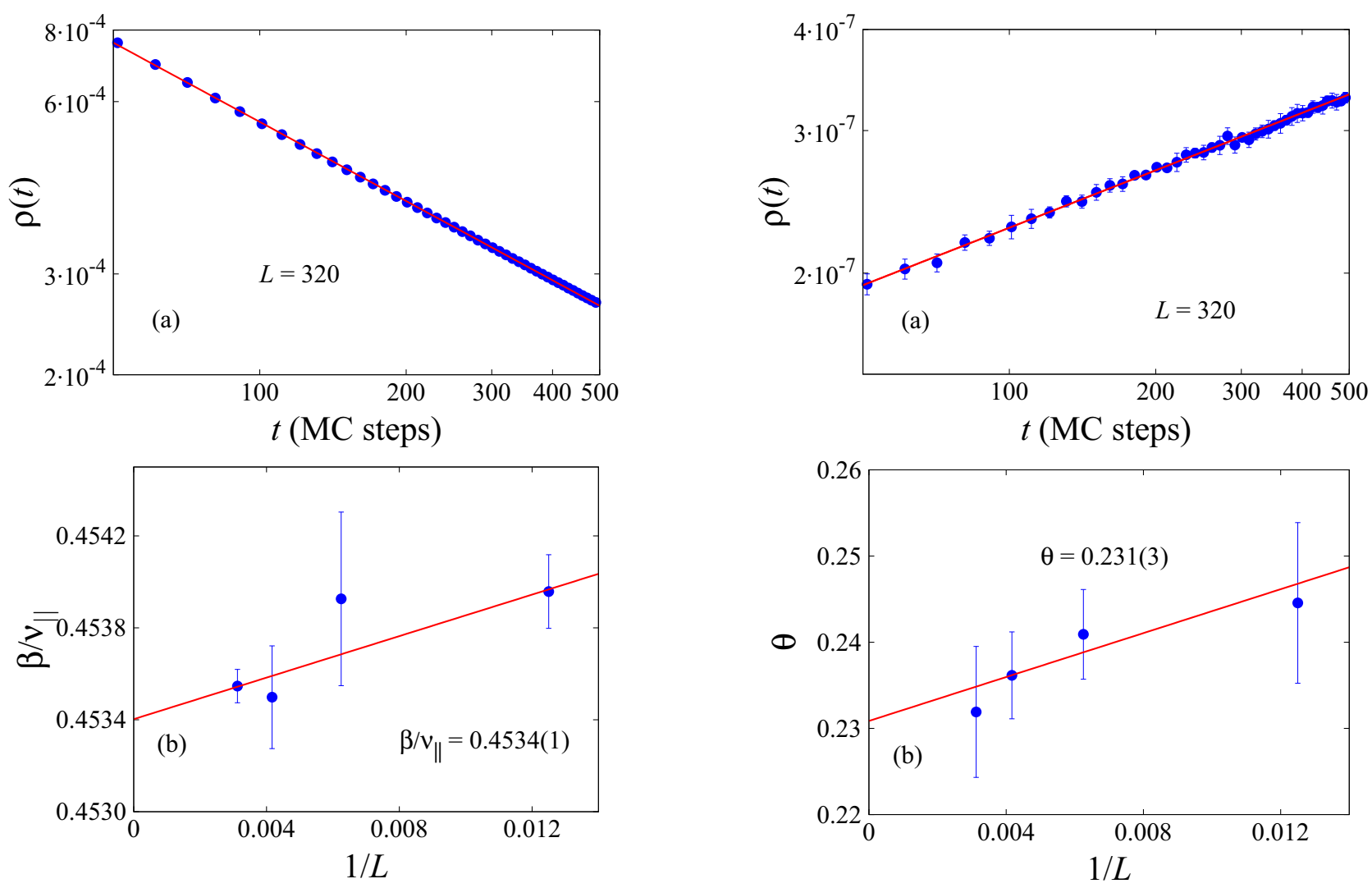

FIG. 5. (a) Time evolution of $\rho(t)$ when the initial lattice is completely empty. (b) Limit procedure $L \rightarrow \infty$ to obtain $\beta / v_{\|}$in the thermodynamic limit.

$\beta / \nu_{\|}$and $\theta$. On the other hand, to obtain the exponent $1 / \nu_{\|}$, we disregarded the $500 \mathrm{MC}$ steps at the beginning of the simulation. In addition, to estimate these exponents with precision, we perform huge simulations with $N_{\mathrm{run}}=10000$ runs.

Figure 5(a) shows the behavior of Eq. (5) in log-log scale for $L=320$. The error bars are smaller then the symbols.

Through the linear fit of this curve we obtain $\beta / \nu_{\|}=$ $0.4535(1)$. In order to take into account the effects of finite size, we also simulate the system with other lattice sizes. In Fig. 5(b), we show the limit procedure $L \rightarrow \infty$ used to reach the thermodynamic limit. In Table II we show our results along with the estimates obtained for $L \rightarrow \infty$.

Figure 6(a) shows the behavior of Eq. (6) in log-log scale for $L=320$. The slope of this curve is the dynamic critical exponent $\theta$.

In Fig. 6(b), we present our estimates for different lattice sizes along with the limit procedure whose result when $L \rightarrow$ $\infty$ is $\theta=0.231(3)$.

TABLE II. Static critical exponent $\beta / v_{\|}$for different lattice sizes as well as the extrapolated value when $L \rightarrow \infty$.

\begin{tabular}{lccccc}
\hline \hline Exponent & $L=80$ & $L=160$ & $L=240$ & $L=320$ & $L \rightarrow \infty$ \\
\hline$\beta / \nu_{\|}$ & $0.4539(2)$ & $0.4539(4)$ & $0.4535(3)$ & $0.4535(1)$ & $0.4534(1)$ \\
\hline \hline
\end{tabular}

TABLE III. Dynamic critical exponent $\theta$ for different lattice sizes as well as the extrapolated value when $L \rightarrow \infty$.

\begin{tabular}{lccccc}
\hline \hline Exponent & $L=80$ & $L=160$ & $L=240$ & $L=320$ & $L \rightarrow \infty$ \\
\hline$\theta$ & $0.245(9)$ & $0.241(5)$ & $0.236(5)$ & $0.232(8)$ & $0.231(3)$ \\
\hline \hline
\end{tabular}



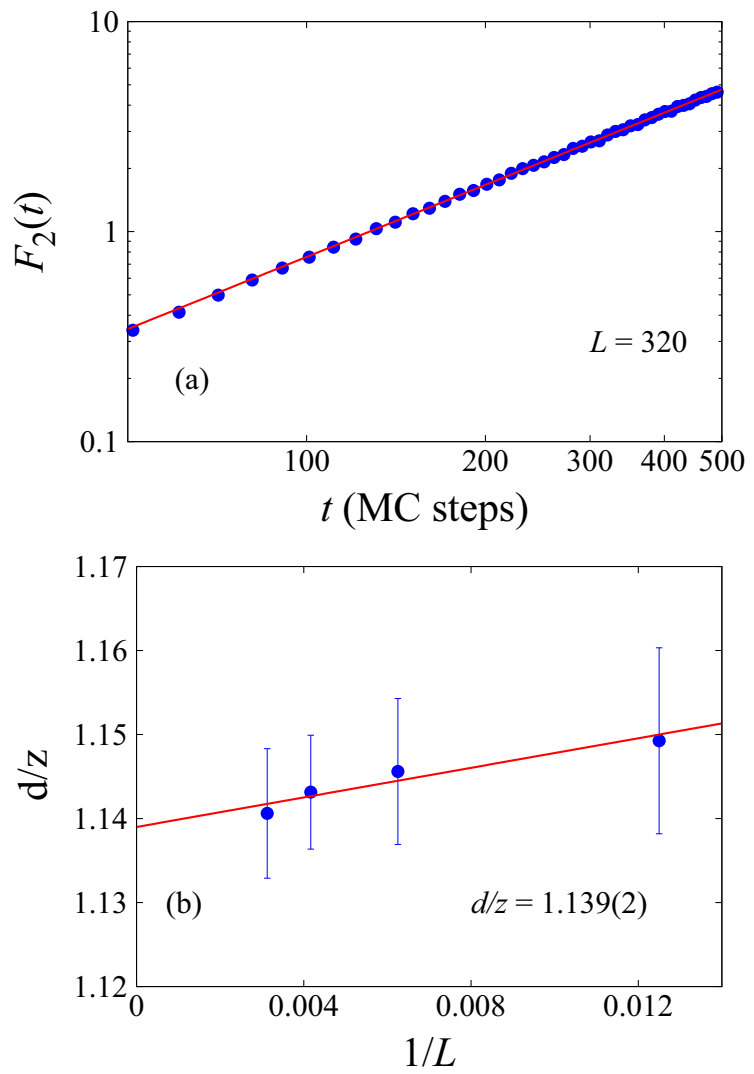

FIG. 7. (a) Time evolution of $F_{2}(t)$ in $\log -\log$ scale for $L=$ 320. The error bars are smaller than the symbols. (b) Limit procedure $L \rightarrow \infty$ to obtain the dynamic exponent $d / z$ in the thermodynamic limit.

In Fig. 8(a) we show the time evolution of $D(t)$ in log-log scale for $L=320$, and the extrapolation is presented in Fig. 8(b). Table $\mathrm{V}$ presents the exponent $1 / v_{\|}$obtained in our simulations for different lattice sizes as well as its extrapolated value.

Finally, with this set of critical exponents in hand, we are able to estimate the static and dynamic critical exponents of the ZGB model independently. Our results, presented in Table VI, are in complete agreement with estimates obtained previously for the model.

These results, along with the localization of the continuous phase transition and the upper spinodal point of the ZGB model, show the efficiency and reliability of short-time Monte Carlo simulations and the coefficient of determination method in the study of systems without a defined Hamiltonian and that possess absorbing states.

TABLE IV. Critical exponent $d / z$ for different lattice sizes as well as the extrapolated value when $L \rightarrow \infty$.

\begin{tabular}{lccccc}
\hline \hline Exponent & $L=80$ & $L=160$ & $L=240$ & $L=320$ & $L \rightarrow \infty$ \\
\hline$d / z$ & $1.149(11)$ & $1.146(9)$ & $1.143(7)$ & $1.141(8)$ & $1.139(2)$ \\
\hline \hline
\end{tabular}
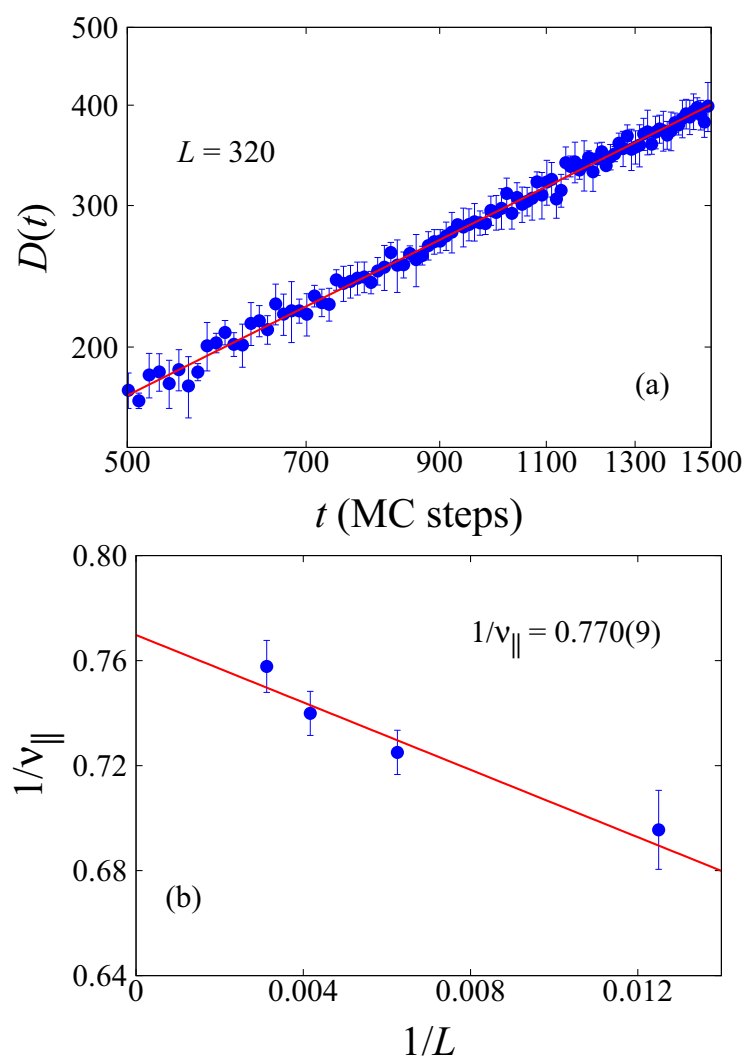

FIG. 8. (a) Time evolution of $D(t)$ in $\log -\log$ scale for $L=320$. (b) Limit procedure $L \rightarrow \infty$ to obtain the static exponent $1 / v_{\|}$in the thermodynamic limit.

\section{CONCLUSIONS}

In this paper we studied the phase transitions of the ZiffGulari-Barshad (ZGB) model by using an alternative method that optimizes the coefficient of determination to localize the critical parameter of the continuous phase transition point and an estimate of the upper spinodal point (one of pseudocritical points) of the weak discontinuous transition point of this model. To obtain these points, we considered the density of $\mathrm{CO}$ molecules $\left(\rho_{\mathrm{CO}}\right)$ and the density of vacant sites $\left(\rho_{V}\right)$ as order parameters of the model. In this study, we found a second peak, on the right side of the upper spinodal point that does not present effects of finite size and therefore was not considered here. However, this point could be the subject of further investigation in order to clarify its meaning and relationship with the discontinuous phase transition of the model. Moreover, we also obtain the critical exponents of the continuous phase transition point by using time-dependent simulations. The exponents $\beta, v_{\|}, v_{\perp}, z$, and $\theta$ were obtained independently from the power laws. Our results are in excellent agreement with previous results. The methodology developed

TABLE V. Critical exponent $1 / v_{\|}$for different lattice sizes as well as the extrapolated value when $L \rightarrow \infty$.

\begin{tabular}{lccccc}
\hline \hline Exponent & $L=80$ & $L=160$ & $L=240$ & $L=320$ & $L \rightarrow \infty$ \\
\hline $1 / v_{\|}$ & $0.696(15)$ & $0.725(8)$ & $0.740(8)$ & $0.758(10)$ & $0.770(9)$ \\
\hline \hline
\end{tabular}


TABLE VI. Static and dynamic critical exponents of the ZGB model.

\begin{tabular}{llc}
\hline \hline Exponent & Our results & Other results [50] \\
\hline$\beta$ & $0.586(7)$ & $0.584(4)$ \\
$\nu_{\|}$ & $1.292(15)$ & $1.295(6)$ \\
$\nu_{\perp}$ & $0.736(10)$ & $0.734(4)$ \\
$\theta$ & $0.231(3)$ & $0.2295(10)$ \\
$z$ & $1.756(3)$ & $1.76(3)$ \\
\hline \hline
\end{tabular}

in this paper can be easily applied to the other surface reaction models by including desorption, impurities, or even mobility of molecules.

\section{ACKNOWLEDGMENT}

This research work was in part supported financially by CNPq (National Council for Scientific and Technological Development under Grants No. 458498/2014-9 and No. 310017/2015-7.)
[1] T. Tomé and M. J. de Oliveira, Stochastic Dynamics and Irreversibility (Springer, Heidelberg, 2015).

[2] H. Hinrichsen, Adv. Phys. 49, 815 (2000).

[3] T. Chou, K. Mallick, and R. K. P. Zia, Rep. Prog. Phys. 74, 116601 (2011).

[4] R. da Silva and N. Alves Jr., Physica A 350, 263 (2005).

[5] L. Ingber, Math. Model. 5, 343 (1984).

[6] R. da Silva, M. Zembrzuski, F. C. Correa, and L. C. Lamb, Physica A 389, 5460 (2010).

[7] C. Castellano, S. Fortunato, and V. Loreto, Rev. Mod. Phys. 81, 591 (2009).

[8] R. da Silva et al., Int. J. Bifurcation Chaos 20, 369 (2010); Phys. Rev. E 88, 022136 (2013); Physica A 437, 139 (2015); R. da Silva, Braz. J. Phys. 38, 74 (2008).

[9] C. G. Rodrigues, A. A. P. Silva, C. A. B. Silva, A. R. Vasconcellos, J. G. Ramos, and R. Luzzi, Braz. J. Phys. 40, 63 (2010).

[10] R. da Silva et al., Philos. Trans. R. Soc. London A 369, 307 (2011); J. Stat. Mech. (2010) P04025; Appl. Math. Model. 34, 968 (2010)

[11] H. Hinrichsen, Braz. J. Phys. 30, 69 (2000).

[12] J. Blease, J. Phys. C 10, 917 (1977); 10, 925 (1977); 10, 3461 (1977).

[13] H. K. Janssen, Z. Phys. B 42, 151 (1981).

[14] P. Grassberger, Z. Phys. B 47, 365 (1982).

[15] J. W. Evans, Langmuir 7, 2514 (1991).

[16] E. V. Albano, Surf. Sci. 306, 240 (1994).

[17] M. F. de Andrade and W. Figueiredo, Phys. Rev. E 81, 021114 (2010).

[18] M. F. de Andrade and W. Figueiredo, J. Chem. Phys. 136, 164502 (2012).

[19] M. Ehsasi, M. Matloch, J. H. Block, K. Christmann, F. S. Rys, and W. Hirschwald, J. Chem. Phys. 91, 4949 (1989).

[20] K. Christmann, Introduction to Surface Physical Chemistry (Steinkopff Verlag, Darmstadt, 1991), p. 1274.

[21] R. Imbhil and G. Ertl, Chem. Rev. 95, 697 (1995).

[22] R. M. Ziff, E. Gulari, and Y. Barshad, Phys. Rev. Lett. 56, 2553 (1986).

[23] I. Jensen, H. C. Fogedby, and R. Dickman, Phys. Rev. A 41, 3411 (1990).

[24] P. Meakin and D. J. Scalapino, J. Chem. Phys. 87, 731 (1987).

[25] R. Dickman, Phys. Rev. A 34, 4246 (1986).

[26] P. Fischer and U. M. Titulaer, Surf. Sci. 221, 409 (1989).

[27] M. Dumont, P. Dufour, B. Sente, and R. Dagonnier, J. Catal. 122, 95 (1990).

[28] E. V. Albano, Appl. Phys. A 55, 226 (1992).
[29] T. Tomé and R. Dickman, Phys. Rev. E 47, 948 (1993).

[30] H. P. Kaukonen and R. M. Nieminen, J. Chem. Phys. 91, 4380 (1989).

[31] I. Jensen and H. C. Fogedby, Phys. Rev. A 42, 1969 (1990).

[32] B. C. S. Grandi and W. Figueiredo, Phys. Rev. E 65, 036135 (2002).

[33] G. L. Hoenicke and W. Figueiredo, Phys. Rev. E 62, 6216 (2000).

[34] G. M. Buendía and P. A. Rikvold, Phys. Rev. E 85, 031143 (2012).

[35] G. M. Buendía and P. A. Rikvold, Phys. Rev. E 88, 012132 (2013).

[36] G. M. Buendía and P. A. Rikvold, Phys. A 424, 217 (2015).

[37] G. L. Hoenicke, M. F. de Andrade, and W. Figueiredo, J. Chem. Phys. 141, 074709 (2014).

[38] J. Satulovsky and E. V. Albano, J. Chem. Phys. 97, 9440 (1992).

[39] E. V. Albano, Surf. Sci. 235, 351 (1990).

[40] B. J. Brosilow, E. Gulari, and R. M. Ziff, J. Chem. Phys. 98, 674 (1993).

[41] J. Marro and R. Dickman, Nonequilibrium Phase Transitions in Lattice Models (Cambridge University Press, Cambridge, 1999).

[42] K. S. Trivedi, Probability and Statistics with Realiability, Queuing, and Computer Science and Applications, 2nd ed. (John Wiley and Sons, Chichester, 2002).

[43] R. da Silva, J. R. Drugowich de Felício, and A. S. Martinez, Phys. Rev. E 85, 066707 (2012).

[44] R. da Silva, N. Alves Jr., and J. R. Drugowich de Felicio, Phys. Rev. E 87, 012131 (2013).

[45] R. da Silva, H. A. Fernandes, J. R. Drugowich de Felício, and W. Figueiredo, Comput. Phys. Commun. 184, 2371 (2013).

[46] R. da Silva, H. A. Fernandes, and J. R. Drugowich de Felício, Phys. Rev. E 90, 042101 (2014).

[47] R. da Silva and H. A. Fernandes, J. Stat. Mech. (2015) P06011 (2015).

[48] J. W. Evans and M. S. Miesch, Phys. Rev. Lett. 66, 833 (1991).

[49] E. V. Albano, Het. Chem. Rev. 3, 389 (1996).

[50] C. A. Voigt and R. M. Ziff, Phys. Rev. E 56, R6241 (1997).

[51] R. M. Ziff and B. J. Brosilow, Phys. Rev. A 46, 4630 (1992).

[52] A. Golchet and J. M. White, J. Catal. 53, 266 (1978).

[53] T. Matsushima, H. Hashimoto, and I. Toyoshima, J. Catal. 58, 303 (1979).

[54] J. H. Block, M. Ehsasi, and V. Gorodetskii, Prog. Surf. Sci. 42, 143 (1993).

[55] M. Berdau, G. G. Yelenin, A. Karpowicz, M. Ehsasi, K. Christmann, and J. H. Block, J. Chem. Phys. 110, 11551 (1999).

[56] G. Grinstein, Z.-W. Lai, and D. A. Browne, Phys. Rev. A 40, 4820 (1989). 
[57] H. K. Janssen, B. Schaub, and B. Schmittmann, Z. Phys. B 73, 539 (1989).

[58] R. da Silva, N. A. Alves, and J. R. Drugowich de Felício, Phys. Lett. A 298, 325 (2002).

[59] H. A. Fernandes, Roberto da Silva, and J. R. Drugowich de Felício, J. Stat. Mech.: Theor. Exp. (2006) P10002.

[60] R. da Silva, R. Dickman, and J. R. Drugowich de Felício, Phys. Rev. E 70, 067701 (2004).
[61] P. Grassberger and Y. Zhang, Physica A 224, 169 (1996).

[62] D. A. Huse, Phys. Rev. B 40, 304 (1989).

[63] L. Schulke and B. Zheng, Phys. Rev. E 62, 7482 (2000).

[64] E. Albano, Phys. Lett. A 288, 73 (2001).

[65] V. S. Leite, G. L. Hoenicke, and W. Figueiredo, Phys. Rev. E 64, 036104 (2001). 